lectual deficiency. The patient is undersized for his years. His face which is pale, is unequal on the two sides; the left half is smaller than the right. He cannot close his mouth properly nor approximate his lip as in the act of whistling. The left angle of the mouth is gaping, and of the brow and slightly to the left of the middle line is an elongated of the brow and slightly to the left of the middle line is an elongated
mark with depression. This, the mother maintains, is the impression left by the blade of the forceps. It is said never to have disappeared from the day on which the patient was born. Beyond simply stating be caused by the forceps I offer no opinion upon this point

In spes by the forceps, I offer no opinion upon this point. compared to the left. The left arm is not only thind ful of expression the right by inch. Part of this may be explained by the arm being kept in a state of flexion. The proximal phalanges of the fingers are extended, whilst the others are flexed towards the palm. He cannot hold out his left hand steadily; when it is unsupported it is the seat of choreiform movements, and by the fingers of this hand delicate movements, such as picking up a pin, are more or less impossible; this is accomplished by a grabbing movement. He is not only unable to control these choreiform movements, but when asked to move certain fingers only he cannot do so, the whole of the fingers are then thrown into a state of irregular flexion and extension. Sensation is perfectly normal. Right arm over biceps measures $6 \frac{1}{2}$ inches and left 6 inches. There is a slight drooping of the left upper eyelid ; the pupils are normal i the discs are healthy. The upper teeth are large and irregular. Heart, lungs, liver, and spleen
are healthy. The left leg remains in a condition of spastic rigidity; the are healthy. The left leg remains in a condition of spastic rigidity; the heel is drawn up; the toes are pointed as in talipes equinus, the big toe developed than those of the right, and the knee-jerk is excessive on both sides. Ankle clonus is not obtainable, but it is observed that the left leg exhibits a series of recurrent small tremors, akin to clonus, withou provocation. The above was the condition of the patient three years ago: been equal to that of another boy of his own age. The knee jerks are not so excessive as they were.

The history of a severe labour, followed shortly afterwards by convulsions in the infant, the weakness and maldevelopment of the left side of the face, left arm and leg, and the irregular, almost athetoid movements exhibited, lead me to believe that the symptoms in our patient can only be explained by conditions which came into existence during the process of his being born. Gowers ${ }^{2}$, in his article on Infantile Meningeal Hæmorrhage, gives a very graphic description of cases such as this. He also states, as the result of his own experience and that of Drs. Little and Sarah McNutt, that meningeal hæmorrhage occurring during parturition inflicts such an injury upon the brain that complete recovery becomes a matter of difficulty. In most of his cases the presentation was unnatural; the head was born last. "Mechanical congestion accompanied the compression, hence the occurrence of hæmorrhage is easy to understand." Whether the child came head or breech foremost there seems to have been in almost every case some difficulty in the process of parturition, and also some difficulty in eliciting signs of life in the child immediately after its birth. Where the forceps has been used and birth palsy has followed, we are scarcely warranted in assuming that the disease is to be attributed to their employment, for the conditions which necessitated their being used were in all probability sufficient to cause the compression upon which we believe the hæmorrhage depends.

Considering the large number of children born that have to be resuscitated by artificial respiration, it is but very few after all who suffer from birth palsy, and in this matter I exclude those cases of facial paralysis in the newly-born infant, which disappear a few days after birth, and which are caused by the pressure of the forceps upon the nerve itself. Given the case of an infant born under circumstances where great compressing influences have been in operation, that child may from the first have convulsions and exhibit rigidity and paralysis, or it may be that for the first few days or weeks nothing is noticed which attracts attention.

CASE II. Precipitate Labour: Meningeal Hæmorrhage ; Death.-In one of my own cases seen with Dr. Wilson, of Wallsend, the labour was, comparatively speaking, easy. It was, perhaps, rather precipitate, and therefore the compression would be sudden, for the whole period of labour scarcely lasted an hour and a-half. The baby was born with very severe pains before the arrival of the doctor. The child was extremely feeble child had in no way been injured after its escape from the vulva. From the first the child's respiration was extremely feeble, and the beat of its heart weak. The child was so prostrate and lifeless that it could scarcely swallow a few drops of liquid. It died on the third day from what appeared to be sheer exhaustion. At the post-mortem examination several hæmorrhages were found under the skin of the occipital region, under the dura mater, over the vertex of brain, and under the tentorium cerebelli. Other portions of the membrane of the cerebellum were extremely
hyperæmic : the brain and cerebellum were healthy, and there was no
effusion into either of the lateral ventricles. In addition, there were numerous small hæmorrhages, varying in size from a pin's head to a pea.

2 Diseases of Nervous System.
The vessels in the pia mater were distended; some had ampullar dilata Then upon them, whilst others could be traced into the efrusions of studded here and there with reddish-black dots of pulmonary apoplexy. These conditions, not unlike those of the early stages of pneumonia. are to be explained by atalectasis due to insufficient respiratory force. The other organs of the body were healthy.

Here then, was a case where meningeal hæmorrhage occurred during parturition without the forceps having been employed, and apparently where no long-continued compressing influences had been at work; and if the child had ived, in addition to mal-development, there would undoubtedly have been the characteristic symptoms of birth palsy.

It is hæmorrhages, such as the one I have described, spread over the vertex and posterior part of the brain that havebeen found by Gowers and others in cases where convulsions, rigidity, and paralysis were present either at birth or shortly after it. Meningeal hæmorrhage occurring under these circumstances upon the vertex either compresses the convolutions of the brain, or it tears the cerebral tissue, or the blood is poured out at the base in the posterior fossa, and surrounds the medulla and cerebellum. Basal hæmorrhage is regarded by McNutt as that most likely to arise when there has been a head presentation, whilst the effusion of blood upon the vertex, on the other hand, is regarded as more likely to develop when it has been a foot presentation. Without accepting this as absolutely true, it is no doubt what would be most likely to happen. In a breech presentation, for example, where all but the head is born, the neck of the child is caught more or less by the muscular ring at the exit of the uterus and the base of the skull being also more or less compressed, there would be impeded escape of blood from the brain, and the vessels at the vertex, thereby inordinately distended, might easily rupture, and the result would be an extravasation of blood upon the surface of the brain. Necropsies made upon infants that have died shortly after birth have demonstrated the existence of this form of meningeal hæmorrhage, and in the case of children that have survived this disaster and died years afterwards, having exhibited during life the characteristic symptoms of birth palsy, post-mortem examination has shown the lesion to have been one of atrophy of the convolutions of the motor area. The appearances presented could only be explained by the suppo; sition of there having been a hæmorrhage which had inflicted injury upon the brain, the blood having been slowly absorbed; leaving, however, considerable damage done to the cortex. The seat of the hæmorrhage, generally upon the vertex, explains the symptoms, namely, paresis of the muscles of the legs and arns. The effusion may extend lower down apon the ascending frontal convolution, and destroy the centres for the muscles of the face, whilst the excessive knee-jerks and rigidity in all cases would be due to descending degeneration of motor tracts, and the spontaneous movements to the per: sistence of damaged nerve cells in the cortex. In $R$. $H$. the disease is unilateral but in many it is bilateral, and where this is the case the paralysis is more marked on one side- of the body than the other, the two limbs most affected being: always on the same side.

CAsE III. Spastic Paraplegia.-A short while ago I saw a boy, aged 5 years in whom the disease had assumed the characters of " spastic paraplegia." Both legs were the seat of clasp-knife rigidity that he could not stand unless supported. Both feet were in a condition of talipes equinus. The foot and leg of one side, owing to the action of the adductor muscles of the thigh, were thrown in front of the other, almost at right angles to it, so that it was impossible for him to stand alone or walk. The legs were fairly well developed as regards length, but the muscles were imperfect, and were rigid. There was spasmodic contraction of the muscles of the calves; knee-jerks were excessive. There was no ankle clonus; noticed in the arms.

\section{ON PERIPHERAL BIRTH PALSY.}

BY WILLIAM GAY, M.D., M.R.C.P.,

Late Clinical Assistant, Great Ormond Street Children's Hospital.

Factar Parst.

THe simplest form of peripheral birth palsy is that which affects the seventh cranial nerve. It is generally the result of forceps operation, but is sometimes present after a protracted, though otherwise normal, labour. The general tendency of such cases is to get rapidly well in the course of a 
ew weeks or months, but I once saw a young woman in whom the paralysis had persisted, and other cases are recorded by Duchenne and Stephan. I lately saw a typical instance of this affection with Dr. Rumboll, of Leeds. The patient was born after a protracted and difficult labour, in which the high forceps operation had been performed. The cranial bones were strongly over-riding one another, and the facial paralysis, at first complete, rapidly improved, so that little or no evidence of it remained at the end of two months. In all these cases the paralysis is evidently due to pressure either of the forceps or maternal structures, and the condition may be compared to pressure paralysis of the musculospiral and other nerves. Stephan suggested that there was a necrosis of the temporal bone in his persisting case of facial paralysis, but of this there was no evidence.

\section{Brachial PaIsy.}

Of much more serious import is peripheral birth palsy of the arm, which has received but scant notice in this country. Evidence of this is to be found in the first number of the Archives of Surgery, where Mr. Jonathan Hutchinson publishes a case of peripheral birth palsy, and expresses his belief that it is the first one recorded. Sporadic cases have been described since 1851 , when Danyan ${ }^{2}$ published a very typical example of the affection. This was followed by Roger's ${ }^{3}$ case in 1864 and Guéniot's ${ }^{4}$ in 1867. To Duchenne, however, belongs the credit of having so fully described the condition that very little addition to our knowledge of it has since been made. Peripheral birth palsy is probably not at all rare. Duchenne mentions that he met with four cases, complicated with dislocation of the shoulder, in the course of a month, and Suckling ${ }^{6}$ that he often saw cases of the affection at the Birmingham Children's Hospital. I have myself come across at least four instances of it, ${ }^{7}$ two of which I will now describe.

CASF I.-A. H., aged 3 months, was brought to the Hospital for Sick youngest of three children. all born at full time (1) died at birth, very large, had to be destroyed; (2), a boy of 2 years, the labour was very tedious, and he had a "dropped" arm for a week; he looks healthy, had thrush, which "went through him," no other evidence of specific disease ; (3) patient, breech presentation, labour twenty-four hours, right inquiry of mine that there was great difficulty in London, replied to an and that " all the children were unusually large at birth." There was no instrumental interference. The right arm was noticed paralysed from the first, and hung quite flaccid. When brought to hospital the shoulder was found morable in all directious, but not quite so freely as the left. The elbow was extended, and there was slight resistance to flexion, which was, however, easily overcome. The wrist was dropped, the fingers ex. tended at the metacarpo-phalangeal joints, and flexed at the inter-phalangeal ; the thumb extended and adducted. There was no very evident wasting, except of the thenar and hypothenar eminences, and the right shoulder was not quite so rounded as the left. Upon careful measurement the right forearm and upper arm were found to be $\frac{1}{8}$ to $\frac{1}{4}$ in. smaller in circumference than the left, and the muscles were much more flabby. The whole palm was markedly flattened; no evidence of paralysis of cervical sympathetic. Electrical examination unsatisfactory on account of screams and struggles of child, but it was evident that there was no response of the muscles of upper arm, forearm, or hand to a faradic current. which caused the muscles of left arm to contract with ease. The whole limb was colder and bluer than the left, and the finger nails (at 5 months) had a distinct transverse ridge separating the distal brownish dead nail from that which was healthy, and composed about two-thirds of the whole. The nails of the left hand were normal in their entire length. Sensation not accurately tested, but faradic current caused greater struggles when applied to left arm. The right face and leg were normal, and knee-jerks present and equal. There was no evidence of any fracture or dislo was subof of syphis. Like the second child, the patient had had thrush, and, in ing, snuffles, sore nates, or craniotabes, and the children were all born at full term.

Treatment at first consisted of inunction with mercury, and rubbing the arm with oil. In January, 1888 , some iodide of potassium (gr. $\mathbf{j}$, ter die) was added. In February it is noted that there has been some slight improvement. The shoulder can be abducted and the elbow a little flexed, so that the patient can almost raise the arm to her mouth. The wrist and fingers are still quite paralysed. April 9th, 1888: Localised faradisation of arm for past month; can move thumb now. The mercury stituted.

$$
\begin{aligned}
& 1 \text { BRITISH MFDical JoURnat, 1888, vol. i, p. } 656 . \\
& 2 \text { Bulletin de la Socisté de Chirur., t. ii, p. } 148 . \\
& 4 \text { Bulletin de la Societe de Chirur., p. 34, } 1867 . \\
& \begin{array}{l}
4 \text { Loc. cit. } \\
5 \text { Lalletin de la Societe de }
\end{array}
\end{aligned}
$$

BRITISH MFdical Journal, vol. ii, 1888, p. 1277 Dr. Abercrombie.
CASE Ir. -M. H., aged 3 months, brought to the Children's Hospital, Great Ormond street, early in 1888. Mother five pregnancies (1) child, now 7 years, and always well and strong ; weight at birth 11 lbs., no forceps, arm dropped, and useless for a week after birth ; $(2,3$, and 4$)$ mis carriages at 3 to 31 ' months ; (5) patient, head presentation, thirteen hours labour, baby very large (not weighed), delivered with forceps, nearly dead, artificial respiration vigorously carried out. Patient much larger paralysed from first day. The midwife told the mother that " the shoulder stuck," and expressed her belief that the arm was then injured by the doctor. Patient had had snuffles but no other evidence of syphilis. Condition when first seen : Right arm paralysed with the exception of the fingers, which have always been freely used. Upper arm slightly rotated inwards, elbow extended, wrist strongly, pronated. The right arm can be severely pinched in places without making the patient cry. Transverse ridge on nails of right fingers, about two-thirds down. The paralysed limb can be made to move freely in all directions and without pain. No fracture or dislocation discoverable ; pupils equal and dilate in shade; no signs of paralysis of cervical sympathetic; face and legs normal; kneejerks active and equal; head falls to right side, is never held up straight:
there is a scar over right eye (?) due to forceps; no scar in neck. Treatment consisted in localised faradisation and rubbing; when last seen the patient had practically completely recovered.

These cases are in a way complementary to one another, and illustrate very well the general features of the affection. They also present some special points of interest. In both there was a transverse ridge on each nail of the affected limb between the proximal healthy and distal diseased part. This is. perhaps, most frequently found after acute febrile conditions; but I have also seen it in three cases of peripheral neuritis, to which peripheral birth palsy is most closely allied. It is not always present in the latter affection, for its absence has been specially noted in some instances. In both of the above-described cases the children were said to have been very large at birth. An abnormal size of the child, especially of the body, is one of the most frequent causes of peripheral birth palsy, and Case II probably illustrates the way in which it happens. After the head is born the shoulder "sticks" on account of the large size of the body, traction is made in the nearest armpit, and the nerves are stretched or otherwise injured. Case I exemplifies another common method of causation.

In a breech or foot presentation an arm gets above the head, and in the efforts made to bring it down by means of a traction hook or the bent finger one or more of the bones may be fractured or dislocated and the nerves injured. Such an accident does not necessarily betray any want of skill on the part of the operator, for one of the recorded cases occurred in the practice of Tarnier himself. In a few rare cases the paralysis resulted from the pressure of a much curved forceps blade, which had gripped a spot in front of the trapezius and so damaged the subjacent nerves. A mark or scar generally remains to indicate the origin of the affection. In two cases in which this accident is recorded death occurred in a few days, probably as a result of the injuries.

Distribction of Peripheral Birth Palsy.

The distribution of the paralysis varies considerably according to the nerves damaged. Sometimes the face is paralysed on the same side as the monoplegia; but in Danyan's case the affection was bilateral. A not uncommon form of paralysis is that which corresponds with Erb's upper arm type, in which the deltoid, after the supra- and infraspinatus, biceps, brachialis anticus, and supinators, are implicated. This would probably result from damage to the roots of the fifth and sixth cervical nerves, though Erb believed that the sixth root only supplied this group of muscles. In other cases the above muscles more or less completely escape, and the paralysis falls chiefly upon the muscles of the forearm and hand, pointing to an affection of the lower roots of the brachial plexus. More frequently, however, most of the roots of the brachial plexus are involved, but some more seriously than others. In such case improvement occurs up to a certain point, but a greater or lesser amount of paralysis persists. The triceps nearly always escapes, probably because its nervous supply is derived from so many roots-fourth to eighth cervical. The sympathetic is very rarely included in the affection.

Seeligmüller ${ }^{8}$ has, however, published two cases in which the usual symptoms of paralysis of the sympathetic were present, namely, retraction of eye, contraction of palpebral fissure, myosis, contraction of pupil to light, but no dilata${ }^{8}$ Berlin. klin. Woch., 1870, p. 130 ; and Deutsch. Archiv f. klin. Med., xx 
tion in the shade or upon cutaneous irritation. In both of his cases the incidence of the paralysis was chiefly upon the muscles of the forearm and hand. There is never any active contracture-that is, a persistent shortening of muscle dependent upon spasm of the fibres and not upon tissue change. The limb nevertheless assumes certain positions on account of the pull of the antagonists of the paralysed muscles. One of the commonest of these is internal rotation of the humerus, chiefly resulting from paralysis of the infra-supinatus and the consequent unantagonised pull of the subscapularis. The elbow is usually extended on account of the frequent affection of the flexors. The wrist is generally pronated because of supinator paralysis, and it is sometimes also dropped. When the small muscles of the hand are affected the palm is flattened out in consequence of wasting of the thenar and hypothenar eminences, the proximal phalanges over-extended, and the two distal flexed from paralysis of the interossei. All these positions assumed by the limb are easily overcome, resulting as they do, not from spasm, but from a physiological condition of tone of certain unantagonised groups of muscles. The electrical examination can only be very incomplete in most of these cases on account of the cries and struggles of the patients. It can usually, however, be shown that the faradic contractility is diminished or gone, and sometimes that there is an increase of voltaic irritability and the reaction of degeneration is obtained. It is very difficult to test the degree and extent of anæsthesia in young children, but it is generally evident that there is some loss of sensation of the paralysed as compared with the healthy limb. Trophic disorders are rare. In a case published by McAldowie ${ }^{\circ}$ it is noted that for five months the affected arm did not grow at all, and the ridged nails of my own cases may be taken as evidence of nutritional disturbance. Vasomotor troubles are more common, for the paralysed parts have frequently a dusky red or bluish colour, and are at the same time colder than normal.

DiAgNosis

The diagnosis is not always so easy as might at first appear. A case may not come under observation until the early history is forgotten, and as at any rate one instance is recorded in which several members of a family were successively affected the absence of such history might lead to the belief that they were suffering from a hereditary form of amyotrophy. In the cerebral variety of birth palsy it is extremely uncommon for a single limb to be implicated, and difficulty can only arise when the arm is the part affected, for, as far as I know, no case has yet been recorded of peripheral birth palsy of the leg. The paralysis of cerebral origin is at one time or another associated with a certain amount of spasm, giving rise to a true contracture, which contrasts with the simple flexion or extension of the joints occurring in peripheral paralysis. Duchenne $^{10}$ has recorded a very interesting case, and one very difficult to explain, in which there was spasm of some of the muscles of the arm associated with atrophy of the interossei and thenar muscles. There is no defect of sensation in cerebral cases, but convulsive seizures of the Jacksonian type may possibly occur. Peripheral birth palsy is not likely to be mistaken for cerebral syphilis or infantile paralysis, since the latter occur only during the later periods of infancy. Parrot's or pseudo-paralysis may occasion some difficulty, though only rarely, for its most frequent date of onset is from the second week to the second month. Porak, 11 Parrot, ${ }^{12}$ and Guénoit ${ }^{13}$ have, however, described cases existing from birth, and as in two of these the paralysis was associated with certain fractures, the likeness to peripheral birth palsy was very close indeed. The points of distinction are these. Pseudo-paralysis is, as the name implies, no paralysis at all, and if the limb be carefully watched movements of some of the distal joints may generally be observed; although too none of the cardinal signs of syphilis are at first present they soon develop, and there is periostitis, separation of epiphyses, or some evidence of syphilis of the long bones. The course of the disease is at first progressive, and its termination is either towards rather rapid recovery under

$$
{ }^{9} \text { Brain, vol. ix, p. } 243 .
$$

11 Soc. de Chirurg.. October 31st, 1877.

12 Archiv. de Physiologie, 1871-2, p. 326.

"' Gaz. des Hôpitaux, February ith, 1869 . treatment or death. In peripheral birth palsy, on the other hand, the paralysis is at first complete, there are not necessarily any signs of syphilis, and the condition is chronic. Besides, the muscles do not respond normally to the faradic current, and the paralysis is limited to one arm and possibly the face of the same side.

Progrosis.

Ross $^{14}$ considers the prognosis unfavourable, but Gowers ${ }^{15}$ asserts that most obstetrical cases recover slowly. The records of such cases as I have been able to consult would seem to show that complete recovery is rare, but that no improvement at all should take place is perhaps equally rare. The rule is that some movements eventually become possible, but, compared with those of the healthy limb, are weak and illsustained, for example, a child whose arm hung pendulous by its side at birth may after a time be able to raise the hand to his mouth. That such cases, which have possibly been under treatment for some months, should completely recover when lost sight of appears very improbable. The retention of faradic contractility, though diminished, shows that the rupture or laceration of the nerve trunks is not complete, and is therefore a favourable prognostic sign.

\section{Treatment.}

Electricity is the only method as yet suggested for the treatment of these cases. This, to be successful, must be commenced at an early stage of the disease, and carried out in a systematic manner. Henoch ${ }^{16}$ truly observes that "the persistent employment of electricity can be of service only 80 long as the nerves have not undergone fatty degeneration and the muscles still react," that is, to faradism. There are chiefly two indications in carrying out the electrical treatment: (a) to play upon the affected nerve roots, and $(b)$ to preserve as far as possible the nutrition of the muscles. The former is best obtained by placing a comparatively large electrode over the supra-clavicular fossa, where the roots of the brachial plexus lie, and another in the armpit. The current to be employed in this area is either the faradic or interrupted voltaic of moderate strength, and the application should be continued for five minutes or more. Each of the affected nerve trunks may be treated in a similar, though less thorough, manner. The second indication is fulfilled by subjecting the paralysed muscles to a galvanic or galvano-faradic current with both poles successively for about five minutes. This treatment should be continued for several weeks.

\section{HEADACHE CONSIDERED IN RELATION TO CERTAIN PROBLEMS IN CEREBRAL PHYSIOLOGY. \\ BX HARRY CAMPBELL, M.D., London.}

HRADACHE may be defined as pain felt in any part situated above the base of the skull. It will be convenient to group together under the head of common sensibility (or, as I shall for convenience symbolise it, "C.S.") all the sensations, painful or ot herwise, which may be therein felt; and we may then speak of headache as due to some perturbation of the C.S. centres belonging to the suprabasal structures of the head. Let me here parenthetically suggest that the C.S. centres of the entire body are not, as is generally supposed, limited to a comparatively small region of the cortex, but that their extent is proportional to the enormous extent of tissues which they "represent."

The chief purpose of this paper is to show that there is a very close connection between the C.S. centres of the head, and those of the special senses. The special sensations (of sight, hearing, etc.) having differentiated from common sensation, the nervous systems of the one have differentiated from that of the other. Hence the special sense nerves and their end-organs have differentiated from the neighbouring nerves of C.S., and the special sense centres in each higher level from the neighbouring C.S. centres. From this we might expect to find the special sense centres in the cortex em.

$$
\begin{aligned}
& 11 \text { Diseases of Nervous System, vol. i, p. } 490 \text {. } \\
& 15 \text { Diseases of Nervous System, vol. i, p. } 78 \text {. } \\
& \text { 16 Diseases of Children (Lou's Library), p. } 93 .
\end{aligned}
$$

